\title{
Sistemas de información en salud en la región mesoamericana
}

\author{
Lina Sofía Palacio-Mejía, D en Est de Pobl, ${ }^{(I)}$ Juan Eugenio Hernández-Ávila, D en C, ${ }^{(I)}$ \\ Aremis Villalobos, $M$ en Dem, ${ }^{(2)}$ María Alejandra Cortés-Ortiz, M en Dem, ${ }^{(1)}$ \\ Marcela Agudelo-Botero, M en Dem, ${ }^{(1)}$ Beatriz Plaza, M en $C{ }^{(3)}$ Grupo técnico.*
}

\begin{abstract}
Palacio-Mejía LS, Hernández-Ávila JE,Villalobos A, Cortés-Ortíz MA,Agudelo-Botero M, Plaza B, Grupo técnico. Sistemas de información en salud en la región mesoamericana. Salud Publica Mex 201 I;53 supl 3:S368-S374.
\end{abstract}

\section{Resumen}

Objetivo. Evaluar y analizar los sistemas de información en salud (SIS) en la región mesoamericana. Material y métodos. Se utilizó el marco conceptual y las herramientas de la Red de la Métrica en Salud (RMS) que evalúa seis componentes de los SIS: recursos, indicadores, fuentes de datos, administración de la información, productos y uso. Resultados. La percepción sobre el desarrollo de los SIS de la región mesoamericana se considera presente pero no adecuada $(57 \%)$, con la mejor percepción para México (75\%) y la menor para El Salvador (4I\%). El componente del SIS menos desarrollado, es decir, el no adecuado, fue el de administración de la información (37\%). Por el contrario, el componente con mejor calificación fue el de indicadores (poco más de 69\%, adecuado). En ninguno de los casos los componentes lograron ser muy adecuados. Conclusión. El nivel de desarrollo de los SIS es heterogéneo entre los países. Es necesario generar mecanismos de cooperación para el intercambio de experiencias exitosas que fomenten una colaboración horizontal y permitan mejorar los SIS actuales para dar sustento a la toma de decisiones y a la evaluación de intervenciones en salud, como los de la iniciativa mesoamericana de la salud.

Palabras clave: sistemas de información; diagnóstico; Mesoamérica
Palacio-Mejía LS, Hernández-Ávila JE,Villalobos A,

Cortés-Ortíz MA,Agudelo-Botero M, Plaza B, Grupo técnico. Health information systems in the Mesoamerican region. Salud Publica Mex 20I I;53 suppl 3:S368-S374.

\begin{abstract}
Objective. To evaluate and analyze health information systems (HIS) in the Mesoamerican Region. Material and methods. The conceptual framework and tools of the Health Metrics Network (NHM) was used. It measures six components of the HIS assessment: resources, indicators, data sources, information management, products and use. Results. In this study we found that the average score of the HIS in the Mesoamerican region was $57 \%$, being the maximum value for Mexico (75\%) and the minimum for $\mathrm{El}$ Salvador $(41 \%)$. The item that had lowest scores was that referring to the Management and Administration, where the average assessment was $37 \%$, placing it as present but not adequate. The component with the highest score was Information Products with more than $69 \%$, adequate. In any case, no items were very adequate. Conclusion. The performance of HIS is heterogeneous between countries. It is necessary to strengthen and standardize the criteria of the HIS in the region, so that these are integrated and used in the decision making process based on real information.
\end{abstract}

Keys words: information system; assessment; Mesoamerica

\footnotetext{
* Belice: Roberto Guerra, Ministry of Health, Marylin Pinelo, Statistical Institute; Costa Rica: María Ethel Trejos-Solórzano, Gerardo Solano, Ministerio de Salud; Olga Araya-Umaya, Roberto Dinarte, Instituto Nacional de Estadística y Censos; El Salvador: Marlene Barrientos, Reina Hernández-Santamaría, Ministerio de Salud, William Franklin Sánchez-Orellana, Instituto Nacional de Estadística y Censos de la República; Honduras: María Georgina Díaz, Secretaría de Salud de Honduras; Nicaragua:Alejandro Solís-Martínez, Luis Carballo-Palma, Ministerio de Salud; Panamá: Lizbeth de Benitez, Ilka Tejeda, Ministerio de Salud; Elena Aguilar, Instituto Nacional de Estadística y Censo, Markela Castro, consultora independiente.
}

(I) Centro de Información para Decisiones en Salud Pública, Instituto Nacional de Salud Pública. Cuernavaca, Morelos, México

(2) Centro de Investigación en Salud Poblacional, Instituto Nacional de Salud Pública. Cuernavaca, Morelos, México

(3) Measure Evaluation Proyect de la Universidad de Carolina del Norte. EUA

Fecha de recibido: I5 de marzo de 201 I - Fecha de aceptado: I I de abril de 20I I

Autor de correspondencia: Dr. Juan Eugenio Hernández Ávila, director adjunto del Centro de Información para Decisiones en Salud Pública, Instituto Nacional de Salud Pública.Av. Universidad 655, col. Santa María Ahuacatitlán. 62100 Cuernavaca, Morelos, México. Correo electrónico: juan_eugenio@insp.mx 
L os sistemas de información en salud (SIS) se definen, de acuerdo con la Organización Mundial de la Salud (OMS), como "un mecanismo para la recolección, procesamiento, análisis y transmisión de la información que se requiere para la organización y el funcionamiento de los servicios sanitarios y también para la investigación y la docencia". ${ }^{1}$ El objetivo central de los SIS no debe restringirse a la obtención de datos de problemas específicos de la salud, sino que debe ser una herramienta sistemática y organizada con la que sea posible generar información para el mejoramiento de la gestión y la toma de decisiones en los distintos niveles organizativos de los sistemas de salud. ${ }^{2-5}$ En este sentido, los SIS deben proveer información para la focalización y medición de intervenciones, como las de la Iniciativa Mesoamericana de la Salud (IMS). La IMS tiene como objetivo mejorar las condiciones de salud de la población de los países que integran la región mesoamericana en cuatro temas prioritarios: nutrición, vacunación, enfermedades transmitidas por vectores y salud materna y reproductiva. Esta iniciativa pretende cumplir con su objetivo por medio de la coordinación y desarrollo de actividades de cooperación multilateral que, a su vez, permitan el fortalecimiento de las capacidades regionales de los gobiernos, la coordinación técnica y la implementación de los sistemas de información en salud.

Los SIS son un importante elemento de entrada para la identificación de necesidades, grupos vulnerables, políticas y recursos humanos y financieros, para que, de este modo, se acreciente la capacidad de respuesta a las expectativas de la población y asegure la equidad de la inversión financiera. ${ }^{6}$ Sin embargo, lograr estos propósitos plantea enormes retos organizativos y el compromiso articulado y multidisciplinario de las partes implicadas en el sistema de salud, en los ámbitos local, regional y central. ${ }^{1,2}$

Un SIS debe ofrecer información para las distintas necesidades en la toma de decisiones, tanto en el ámbito individual, como en la elaboración de estadísticas que soporten el desarrollo y evaluación de programas y la formulación de políticas de salud. Los indicadores de salud deben permitir la medición de cambios en tres dominios principales: ${ }^{7}$ los determinantes de la salud, el sistema de salud y el estado de salud de la población. La información que alimentan los SIS proviene de varios subsistemas que pueden operar de forma independiente entre sí, incluso fuera del sector salud; entre ellos, se encuentran los sistemas de vigilancia y notificación de brotes, los datos generados a través de encuestas de hogares, los registros vitales (nacimientos, defunciones y causas de muerte), la recopilación de datos sobre la base de los pacientes y registros de servicios e información de los trabajadores de salud (comunitarios, trabajadores sanitarios y los centros de salud), programas específicos de seguimiento y evaluación (tuberculosis, VIH/SIDA, entre otros), administración y manejo de los recursos (presupuesto, personal y suministros). ${ }^{2,6-9}$

El desempeño de los SIS es heterogéneo entre los países de la región, lo cual implica que las necesidades de información sean abordadas y cubiertas con estrategias diferenciales. Algunos SIS se han visto orillados a desarrollar subsistemas independientes -por presiones económicas-, con objetivos específicos, de acuerdo con las prioridades de las agencias financiadoras; esto ha ocasionado un desarrollo fragmentado del sistema nacional de información en salud..$^{10}$

En este artículo se presenta un diagnóstico y análisis de los SIS en algunos países de la región mesoamericana (Belice, Costa Rica, El Salvador, Honduras, Nicaragua, México y Panamá), en donde se evaluó información acerca de los recursos, indicadores, fuentes de datos, administración de datos, productos de la información, diseminación y uso de la información en salud. Es preciso indicar que en este estudio no se incluyó Guatemala, a pesar de pertenecer a la región, ya que en este país se comenzó con el proyecto de evaluación de los SIS, pero hasta el momento del análisis, no se había concluido.

\section{Material y métodos}

Los resultados contenidos en este documento se basan en información recabada para el proyecto de la IMS, bajo la coordinación del grupo de trabajo de SIS del Instituto Nacional de Salud Pública (INSP), cuya orientación estuvo dirigida a desarrollar un plan de mejoramiento integral de los SIS en la región. La primera etapa fue la elaboración de un análisis integral de los SIS de la región mesoamericana, que se configuró a partir de los diagnósticos de cada país, los cuales fueron realizados utilizando el marco conceptual y las herramientas de la Red de la Métrica en Salud (RMS) entre 2006-2009, y se llevó a cabo con el financiamiento y apoyo técnico de la RMS, la Organización Panamericana de la Salud (OPS), la Agencia de Estados Unidos para el Desarrollo-Oficina para América Latinoamérica y el Caribe (USAID-LAC, por sus siglas en inglés), MEASURE Evaluation, y la IMS.

Los hallazgos obtenidos se discutieron y consensuaron en tres reuniones internacionales en México, Nicaragua y Costa Rica, durante el año 2009, con los expertos de los SIS de los ministerios de Salud y los institutos nacionales de estadística de los países de la región. La RMS es una alianza mundial impulsada por la OMS que "trabaja para aumentar la disponibilidad y utilización de información en salud oportuna y fiable 
en los países en desarrollo y a nivel mundial". ${ }^{11}$ Dicha Red utiliza un marco conceptual que reúne los componentes de oferta y demanda de la información en salud y, por lo tanto, ayuda a definir los sistemas, estándares, capacidades y procesos en el ámbito nacional y mundial, necesarios para fortalecer los SIS.

El diagnóstico de los SIS que se presenta permite obtener una visión sobre los diferentes subsistemas de información estadística de cada país y en general de la región, y da a conocer las percepciones de los gerentes y otros actores clave de la organización respecto a las funciones de los SIS. Para todos ellos, se analizaron seis componentes:

1. Recursos. Incluye el marco legal y regulador para asegurar un funcionamiento total y los recursos disponibles del SIS, incluyendo los recursos humanos, infraestructura, tecnología de la información y comunicación, y mecanismos de coordinación.

2. Indicadores. Evalúa un grupo básico de indicadores que describen las principales áreas de la información en salud (determinantes, recursos y productos del sistema; cobertura de los servicios, calidad y estado), son la base para establecer un plan y una estrategia para un sistema de información en salud.

3. Fuentes de datos. Considera dos tipos: aquellos que generan estimaciones poblacionales (censos, estadísticas vitales y encuestas a hogares), y los que se relacionan con la prestación de los servicios y registros administrativos (vigilancia de las enfermedades, registros de los servicios otorgados por los centros de salud, registros administrativos y encuestas de los centros, infraestructura). Para cada fuente se describe un grupo básico de estándares y estrategias clave.

4. Administración de la información. Incluye los aspectos del manejo de datos: recolección, administración, procesamiento y análisis.

5. Productos de la información. Se refiere a la información misma derivada de los SIS, como indicadores del estado de salud (mortalidad, morbilidad), del sistema de salud, sobre los factores de riesgo, y enfermedades de vigilancia en un formato que pueda ser distribuido y consumido por los tomadores de decisiones (impreso o electrónico). El valor de la información en salud aumenta en la manera en que esté disponible y accesible para los tomadores de decisiones

6. Difusión y uso. Mide aspectos relacionados con el uso de la información en la toma de decisiones en los diferentes niveles de organización del sistema de salud, así como la medida en que se dé importancia a los aspectos organizativos y de comportamiento que limiten su uso.

La herramienta se aplica mediante la técnica de grupo focal. Los participantes de los talleres fueron expertos e informantes clave, constituidos por usuarios y productores de la información en los ámbitos nacional, regional y/o estatal, de las instituciones rectoras y productoras de información en salud como el Ministerio de Salud, el Seguro Social, el Instituto de Estadísticas y los prestadores de servicios públicos y privados de cada país. En total, se abordaron 244 preguntas, las cuales recibieron un puntaje en valores ordinales (0-3) y luego se transformaron en índices que resumen la situación de la categoría bajo evaluación. Los valores tienen la siguiente equivalencia:

Quintil Categoría Color

$10(<20 \%)$

$20(20-40 \%)$

$30(40-60 \%)$

$40(60-80 \%)$

$50(>=80 \%)$

No funcional
No adecuado
Presente, pero no adecuado
Adecuado
Muy adecuado

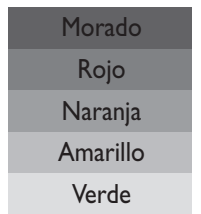

El valor final se obtuvo al calcularse un promedio entre la sumatoria de los puntajes y el número de participantes que evaluaban cada categoría.

\section{Resultados}

\section{Evaluación de los sistemas de información en salud}

Los SIS se percibieron con un desarrollo en promedio de $57 \%$ en la región mesoamericana, con una apreciación mejor en México (75\%) y menor para el caso de El Salvador $(41 \%)$. El rubro que resultó con una menor valoración fue el referente a la administración de la información (37\%), lo que lo sitúa como presente pero no adecuado. Por el contrario, los componentes con mejores calificaciones fueron el de indicadores (69\%) y productos de información $(68 \%)$, catalogados como adecuados. Asimismo, se observan diferencias en los porcentajes obtenidos para cada subcomponente, considerados en el interior de ellos. Las múltiples categorías fueron estimadas en rangos de adecuado y presente pero no adecuado, con excepción de México, donde el componente de productos de información se consideró como muy adecuada, y Nicaragua, donde la administración de la información fue catalogada como no adecuada (cuadro I). 


\section{Brechas por componentes entre países mesoamericanos}

\section{Recursos}

Los recursos, se midieron en tres aspectos: a) políticas y planificación, b) instituciones y recursos humanos, y c) financiamiento e infraestructura de los sistemas de información. En este sentido, se encontró que Costa Rica y México fueron los países que dieron la valoración más alta a este componente, con una clasificación adecuada (60-80\%); Belice, Honduras, Nicaragua y Panamá tuvieron una valoración presente pero no adecuada (40\%-60\%), y El Salvador fue el único que consideró este ítem como no adecuado (20-40\%) (figura 1).

\section{Indicadores}

Los indicadores fueron el segundo aspecto mejor evaluado en todos los países (promedio de 69\%), con valores que oscilan en un rango de 52\% (El Salvador) y 83\% (México). Los indicadores evaluados en este grupo fueron: mortalidad materna e infantil, morbilidad general, morbilidad por tuberculosis, bajo peso en niños, obesidad en adultos, cobertura de vacunación contra el sarampión, asistencia calificada del parto, tratamiento de la tuberculosis, gasto público en salud, gasto privado en salud y densidad de la fuerza de trabajo.

\section{Fuente de datos}

En cuanto a las fuentes de datos, los puntajes van desde 45\% (El Salvador) hasta 75\% (México). Fue evidente que los censos fueron los más altamente calificados por los países como Costa Rica (100\%), Panamá (82\%) y México (81\%), que los consideran como muy adecuados. Asimismo, en tres de los siete países (Panamá, México y Costa Rica) las estadísticas vitales fueron la fuente de información mejor evaluada $(90,91$ y $97 \%$ respectivamente). Otras fuentes bien ponderadas fueron

Cuadro I

EvaluACIÓN DE LOS SISTEMAS DE INFORMACIÓN EN SALUD

\begin{tabular}{|c|c|c|c|c|c|c|c|c|}
\hline \multirow[t]{2}{*}{ Componente } & \multicolumn{8}{|c|}{ Países (\%) } \\
\hline & Belice & Costa Rica & El Salvador & Honduras & México & Nicaragua & Panamá & $R M$ \\
\hline Recursos & 45 & 72 & 36 & 46 & 64 & 43 & 50 & 51 \\
\hline Indicadores & 74 & 58 & 52 & 71 & 83 & 70 & 73 & 69 \\
\hline Fuente de datos & 54 & 74 & 45 & 61 & 75 & 64 & 67 & 63 \\
\hline Administración & 29 & 24 & 25 & 42 & 74 & 18 & 50 & 37 \\
\hline Productos de información & 66 & 74 & 47 & 63 & 84 & 76 & ND* & 68 \\
\hline Difusión y uso & 41 & 38 & 41 & 60 & 71 & 77 & 64 & 56 \\
\hline Promedio/ país & 52 & 57 & 41 & 57 & 75 & 58 & 61 & 57 \\
\hline
\end{tabular}

* Información no disponible

Fuente: elaboración con base en los diagnósticos de los SIS de cada país

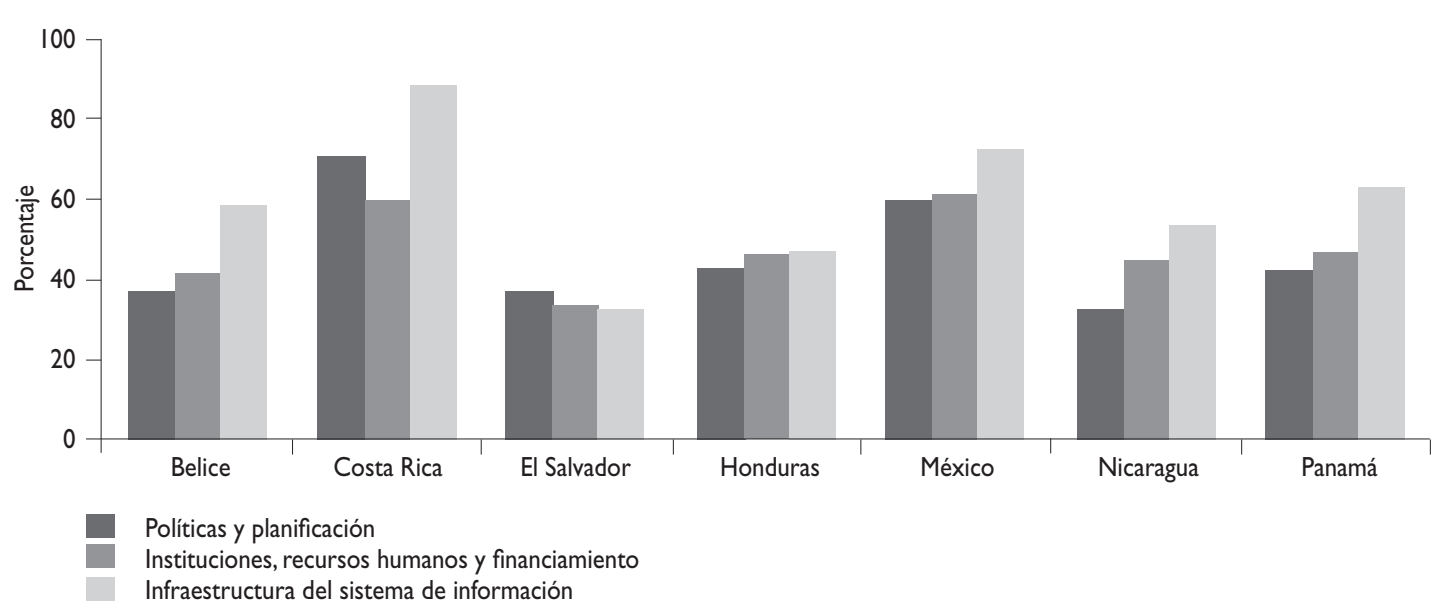

Fuente: Elaboración propia con base en los diagnósticos de los SIS de cada país

Figura I. Evaluación de los RECURSOS EN lOS SISTEMAS DE INFORMACIÓn EN SALUd EN LA REGIÓN MESOAMERICANA 
las encuestas poblacionales. En contraste, los registros administrativos cayeron casi en su totalidad en el grupo de presente pero no adecuados (figura 2).

\section{Administración de datos}

La administración de datos se refiere a aquellos procesos óptimos necesarios para recopilar, compartir y almacenar datos, así como los flujos de información. De acuerdo con el análisis, Nicaragua la consideró como no funcional (18\%); Costa Rica, Belice y El Salvador lo circunscribieron como no adecuado (24, 25 y 29\%); Panamá y Honduras lo encontraron presente pero no adecuado (50 y 42\%), y México lo clasificó como adecuado (74 por ciento).

\section{Productos de información}

Como ya se constató, la producción de información en salud en el marco de los SIS en Mesoamérica es uno de los componentes mejor evaluados $(68 \%)$. No obstante, países como El Salvador lo consideraron bajo en algunas categorías como en los indicadores de factores de riesgo $(24 \%)$ y los indicadores del sistema de salud (36\%). Resalta, entre otras cosas, los valores otorgados por Costa Rica (88\%) y México (89\%), que tuvieron una mejor apreciación de este último rubro mencionado (cuadro II).

\section{Difusión y uso de la información}

La difusión y uso de la información es un elemento considerado en la región como presente pero no adecuado. Al desagregar los datos por subcategorías, el uso de información para la asignación de recursos tuvo la calificación más baja (54\%) y el análisis y uso de la información, la más alta (62\%). Honduras, México y Nicaragua realizaron las evaluaciones más destacadas de Mesoamérica, al catalogar este aspecto como adecuado. El desempeño de El Salvador fue considerado por los expertos con una baja calificación, como se dio para otros puntos ya analizados (figura 3).

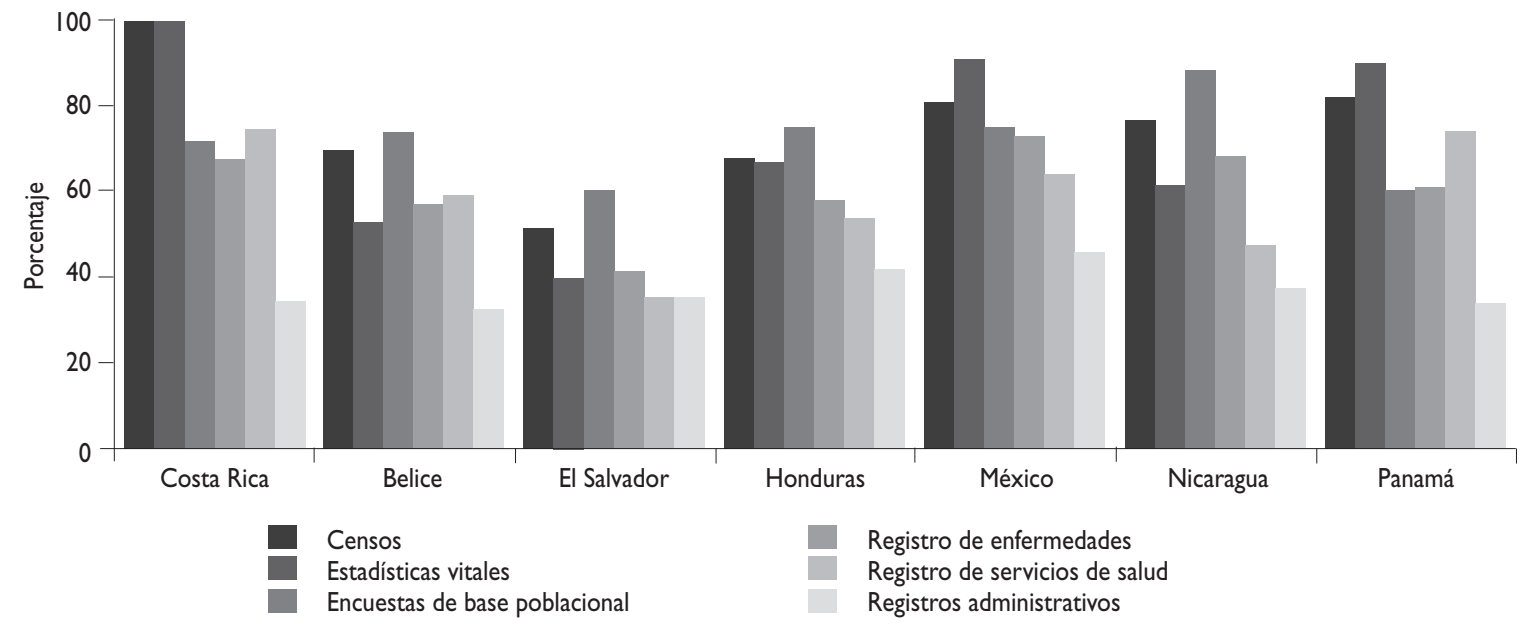

Fuente: Elaboración propia con base en los diagnósticos de los SIS de cada país

Figura 2. Evaluación de LAS fuentes de datos en SALUd EN LA Región Mesoamericana

\section{Cuadro II}

\section{Evaluación DE LA PRODUCCIÓN DE DATOS EN SALUD}

\begin{tabular}{lcccccccc} 
& \multicolumn{9}{c}{ Países (\%) } \\
\cline { 2 - 9 } Componente & Belice & Costa Rica & El Salvador & Honduras & México & Nicaragua & Panamá & RM \\
Indicadores del estado de salud & 77 & 88 & 47 & ND* & 89 & 82 & ND* \\
\hline Mortalidad & 76 & 96 & ND* & 72 & 96 & 77 & 86 & 84 \\
\hline Morbilidad & 79 & 81 & ND* & ND* & 85 & 86 & ND* & 83 \\
\hline Indicadores del sistema de salud & 62 & 62 & 36 & 58 & 74 & 77 & ND* & 62 \\
\hline Indicadores de factores de riesgo & 67 & 93 & 24 & 45 & 76 & 64 & ND*
\end{tabular}

* Información no disponible

Fuente: elaboración propia con base en los diagnósticos de los SIS de cada país 


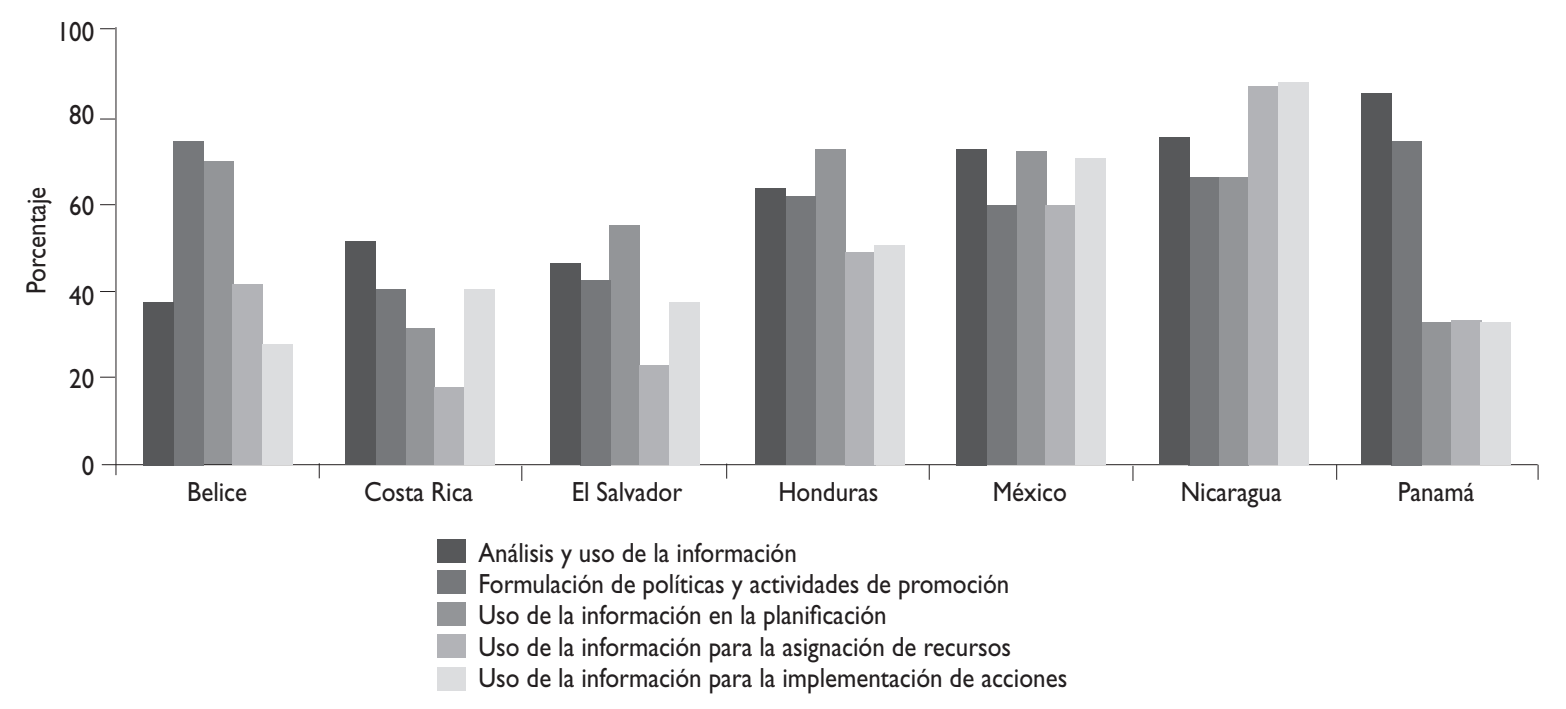

Fuente: Elaboración propia con base en los diagnósticos de los SIS de cada país

Figura 3. Difusión y uso de datos en los SISTEMAS de INFORMACIÓN EN SALUd EN LA REGIÓN MESOAMERICANA

\section{Discusión}

El análisis de los SIS en Mesoamérica se hizo a partir de la aplicación de los instrumentos de la RMS propuesta por la OMS (2006). Si bien esta técnica no está libre de sesgos propios, derivados principalmente por la valoración subjetiva de la percepción de los participantes sobre cada componente de los sistemas de información, es un primer acercamiento para medirlos en forma estandarizada para todos los países de la región.

A grandes rasgos, se hizo una apreciación heterogénea de los SIS entre los países de la región. De esta forma, se encontraron variaciones en aspectos tales como cobertura, calidad y desagregación de la información necesaria, que permiten la identificación adecuada de las intervenciones a realizar, de acuerdo con la población objetivo.

En ningún país se alcanza un SIS adecuado, pero se considera que existe una base sobre la que se puede trabajar para fortalecer los sistemas y tener una información más adecuada. Existen países como Costa Rica y México que obtuvieron una mejor valoración, y países como El Salvador, Honduras o Nicaragua con un menor desempeño; sin embargo, esto sugiere una brecha de oportunidad para trabajar por medio de la cooperación regional, lo que les permitiría intercambiar experiencias exitosas en la implementación y operacionalización de los SIS, con el fin de procurar un nivel más homogéneo en la región.
En general, se encontró que faltan herramientas, métodos, lineamientos y conocimiento de mejores prácticas para la organización y gestión de los SIS, así como compilación, revisión y análisis propio de la información. De este modo, para mejorar la política y planificación, es fundamental adecuar y actualizar el marco legal en ciertos países frente a los SIS, como en el caso de Honduras, Belice, El Salvador y México. Estos países pueden compartir y revisar los marcos legales entre sí e identificar la política y planificación más adecuada de acuerdo con sus necesidades y realidades, que permitan el intercambio adecuado y generalizado de la información. ${ }^{1-6,12-19}$

De igual importancia resulta la administración de los datos, componente con menor desarrollo en la región, por lo que es necesario minimizar las incompatibilidades entre sistemas y recursos tecnológicos en la mayoría de los países mesoamericanos, con el fin de reducir la fragmentación del sistema, todo ello acompañado de la dotación de equipos, mejoramiento de las bases de datos, disponibilidad de plataformas tecnológicas (como el internet y los sistemas de georreferenciación) y el desarrollo de un repositorio o banco de datos interrelacionados, con lo que se lograría la interoperación de diversos usuarios y del expediente médico electrónico. ${ }^{20}$ Previamente, sería indispensable valorar las necesidades de información en distintos niveles técnicos y operativos (tanto en el país como en las localidades), y brindar capacitaciones permanen- 
tes para el personal involucrado con los sistemas de información.

En cuanto al análisis y uso de la información, se observó un uso ocasional de la información dedicada a la planeación y el establecimiento de prioridades, así como una baja cultura en el uso de información para la toma de decisiones en la mayoría de los países. En este sentido, se recomienda que las bases de datos estén disponibles para el público en general de forma amigable y aumentar las estrategias para el análisis de la información y su uso en la toma de decisiones.

En el caso particular de este estudio, la evaluación contribuyó a identificar aspectos integrales de los SIS que aporten a la IM en información para la selección de la población objetivo o medición de las intervenciones en sus cuatro temas prioritarios mencionados (vacunación, vectores, nutrición y salud materna, reproductiva y neonatal) en la región, lo que servirá para la focalización de recursos y el seguimiento posterior a los indicadores generados. Finalmente, hay que señalar que cada país tiene sus propias limitaciones y requerimientos, de ahí que este diagnóstico deba tomarse como punto de partida para el establecimiento de acciones concretas en los SIS y como un referente para el mejoramiento continuo.

\section{Agradecimientos}

El financiamiento para este proyecto fue proporcionado por la Fundación Bill y Melinda Gates vía el Public Health Institute.

Declaración de conflicto de intereses: Los autores declararon no tener conflicto de intereses.

\section{Referencias}

I.World Health Organization. Health information systems development and strengthening: guidance on needs assessment for national health information systems. Geneva: WHO, 2000.

2.Alazraqui M, Mota E, Spinelli H. Sistemas de información en salud: de sistemas cerrados a la ciudadanía social. Un desafío en la reducción de desigualdades en la gestión local. Cad Saúde Pública 2006;22(I2):2693-2702. 3. Cámpoli M. Sistemas de información en el sector salud: Utopía o realidad [sitio de internet]. Fundación Universitaria Dr. René Favaloro (Capital Federal). 2003 [consultado 2010 junio I3]. Disponible en: http:// www.gestiopolis.com/canales $5 / \mathrm{mkt} /$ simsalud.htm.

4. De la Fuente JR, Tapia R, Lezana M. La información en salud. Rev Panam Salud Publica 2002; I2(2): I44-I46.
5. Caja Costarricense del Seguro Social. Sistemas de información en salud en el primer nivel de atención [sitio de internet]. Octava Unidad Modular 2004. [consultado 2010 junio 10]. Disponible en: http://www.cendeisss. sa.cr/cursos/octavaunidad.pdf.

6. Murray C, Frenk J.A framework for assessing the performance. Bulletin of the World Health Organization 2000;79(6):717-732.

7. World Health Organization. Improving the use of information for health care decision-making: what is needed [sitio de internet], 2005 [consultado 2010 junio 14]. Disponible en: http://www.who.int/ healthmetrics/library/issue_I_05apr.doc.

8. World Health Organization. Does South Africa's health information system lead to informed decision-making? [sitio de internet], 2004 [consultado 2010 junio 14]. Disponible en: http://www.who.int/ healthmetrics/library/en/south_africa_05apr.doc.

9. Ashraf $\mathrm{H}$. Countries need better information to receive development AID. Bull World Health Organ 2005;83(8): 10.

10. Vidaurre M, Martínez R, Castillo C. Red de la Métrica de Salud: una alianza mundial para mejorar el acceso a la información para los prestadores de atención en salud y para los encargados de la formulación de políticas. Bulletin of the World Health Organization 2005;26(2):I-7.

II. World Health Organization. Better country data needed to assess progress towards health MDG target. [sitio de internet], 2010 [Consultado 2010 junio 17]. Disponible en: http://www.who.int/ healthmetrics/en/.

12.AbouZahr C, Boerma T. Health information systems: the foundations of public health. Bull World Health Organ 2005;83(8): 10.

13. Murray C, Lopez A,Wibulpolprasert S. Monitoring global health:Time for new solutions. BMJ 2004;329:1096-I I00.

14. Ebrahim S, Smeeth L. Non-communicable diseases in low and middleincome countries: a priority or a distraction? International Journal of Epidemiology 2005;34(5):961-966.

15. Jacucci E, Shaw V, Braa J. Standardization of Health Information Systems in South Africa:The Challenge of Local. Information Technology for Development 2006; 12(3):225-239.

16. Secretaría de Salud de México. Subsecretaría de Innovación y Calidad. Programa de Acción específico 2007-2012. Sistema Nacional de Información en Salud [sitio de internet], 2008 [Consultado 2010 junio I8]. Disponible en: http://www.sinais.salud.gob.mx/descargas/pdf/ PAE2007-2012_SNIS.pdf.

17. Organización Panamericana de la Salud. Diagnóstico del Sistema de Salud de Panamá [sitio de internet]. 2006 [Consultado 2010 junio I8]. Disponible en: http://medicina.uncoma.edu.ar/download/postgrado/ gestion_auditoria/bibliografia/modulo_12/sist_de_informacion_ salud_panama.pdf.

18. Pinet L.Atención prehospitalaria de urgencias en el Distrito Federal: las oportunidades del sistema de salud. Salud Publica Mex 2005;47(I):64-7I. 19. Consejo de Ministros de Salud en Centroamérica y República Dominicana. Plan de Salud de Centro América y República Dominicana 2010-2015. Unidos por la salud de nuestros pueblos. San José de Costa Rica: COMISCA, 2010.

20. Organización Panamericana de la Salud/ Organización Mundial de la Salud. $48^{\circ}$ Consejo Directo. $60^{\circ}$ Sesión del Comité Regional. Punto 4.5 del orden del día provisional. Resolución CD48/9: Plan de Acción Regional para el Fortalecimiento de las Estadísticas Vitales y de Salud [sitio de internet], 2008 [consultado 2010 junio 19]. Disponible en: www.paho. org/english/gov/cd/cd48-09-e.pdf. 